\title{
Bioinformatic studies of vertebrate enolases: multifunctional genes and proteins
}

This article was published in the following Dove Press journal:

Open Access Bioinformatics

8 February 20II

Number of times this article has been viewed

\section{Roger S Holmes}

School of Biomolecular and Physical Sciences, Griffith University, Nathan, QLD, Australia
Correspondence: Roger S Holmes School of Biomolecular and Physical Sciences, Griffith University, Nathan, 4 I I I

QLD, Australia

Tel +6I 737355077

Fax +6I 737357773

Email r.holmes@griffith.edu.au
Abstract: Enolase (ENO) genes and proteins (ENO; EC 4.2.1.11) serve multiple functions in the body, including catalyzing 2-phospho-D-glycerate hydro-lyase activity in glycolysis, assisting hypoxia tolerance, tumor suppression, plasminogen and DNA binding, and acting as a lens crystallin. Comparative ENO amino acid sequences and structures and ENO gene locations were examined using data from several vertebrate genome projects. Vertebrate ENO1, ENO2, and ENO3 genes usually contained 11 coding exons, while ENO4 (encoding an ENO-like protein, ENOLL) usually contained 14 coding exons. Vertebrate ENOF1 (or ENO5) genes encode an antisense RNA, which may regulate mitochondrial thymidylate synthase activity that contained 12-15 coding exons. Vertebrate ENO1, ENO2, and ENO3 sequences shared 78\%-98\% identities but only 19\%-24\% with ENO4 and $>10 \%$ predicted sequence identities with vertebrate ENOF1. Sequence alignments, key amino acid residues, and conserved predicted secondary and tertiary structures were examined, including active site residues (absent in ENO4 and ENOF1) and sites for $\mathrm{Mg}^{2+}$ and plasminogen binding and for acetylation and phosphorylation. The predicted ENO4 structure contained three N-terminal $\alpha$-helices, two $\beta$-sheets, a poly-proline segment, and an extended $\mathrm{C}$-terminal sequence in addition to the typical $\alpha / \beta$ barrel structure reported for ENO1-3 sequences. Potential transcription factor binding sites (TFBS) and CpG islands for regulating ENO gene expression were identified. Human ENO1, ENO2, ENO3, and ENOF1 genes each contained $\mathrm{CpG}$ islands in the gene promoter regions consistent with higher-thanaverage levels of expression. Human ENO3 and ENO1 gene promoters also contained a diverse range of TFBS. The ENO4 gene promoter comprised a $\mathrm{CpG}$ island and several TFBS, including AHR1 in the 5'-UTR region, which may suggest a role for ENO4 in aryl hydrocarbon ligand binding or metabolism. Phylogeny studies of vertebrate ENO1, ENO2, and ENO3 genes and enzymes suggested that they originated in a vertebrate ancestor from gene duplication events of an ancestral ENO1-like gene $>500$ million years ago.

Keywords: vertebrate, amino acid sequence, enolase, evolution, bioinformatics

\section{Introduction}

Enolase (ENO; EC 4.2.1.11) genes and proteins serve multiple functions in the body, including catalyzing 2-phospho-D-glycerate hydro-lyase activity in glycolysis ${ }^{1}$ or playing roles in hypoxia tolerance, ${ }^{2}$ tumor suppression, ${ }^{3}$ and cell surface plasminogen binding ${ }^{4}$ or acting as a lens tau-crystallin, ${ }^{5}$ a DNA-binding protein ${ }^{6}$ or a tubulin/microtubule binding protein during myogenesis. ${ }^{7}$ Three major ENO-like genes have been described on the human genome, ENO1, ENO2, and ENO3, which encode the $\alpha$-, $\beta$-, and $\gamma$-subunits, respectively. ${ }^{8-11}$ Two other human $E N O$-like genes have also been reported, ENO4 (or ENOLL) ${ }^{12}$ and ENO5 (also called ENOF1 or ENOSF1), 
originally identified as encoding an antisense transcript to the thymidylate synthase $(T S)$ gene ${ }^{13}$ which may play a role in regulating the TS locus. ${ }^{14}$

Biochemical studies of vertebrate enolases have characterized several dimeric isozymes containing $\alpha$-, $\beta$-, and $\gamma$-subunits, which are differentially but widely distributed in the tissues of the body. ${ }^{1,15}$ ENO3 encodes the $\beta$-subunit and is predominantly expressed in muscle, whereas ENO2 is more restricted to neural tissues (also called neuron-specific enolase or $\gamma$ ), while ENO1 is expressed in virtually all the tissues of the body, including embryonic tissues, and encodes the $\alpha$-subunit. ${ }^{16,17}$ During vertebrate development, major changes occur in the expression of these genes with a switch from $E N O 1 \rightarrow E N O 3$ and a change from $\alpha \alpha$ to $\beta \beta$ in skeletal muscle and a similar switch from ENO1 $\rightarrow$ ENO2 in nervous tissues with an associated change from $\alpha \alpha$ to an $\alpha \gamma$ and $\gamma \gamma$ enolase isozymes. ${ }^{1,18,19}$ Evolutionary studies have shown that DNA sequences encoding the enolase gene family are highly conserved from yeast to mammalian organisms and that the gene duplication events generating the ENO1, ENO2, and ENO3 genes may have predated the appearance of vertebrates. ${ }^{15,20}$

Structural and molecular modeling studies of lobster, ${ }^{21}$ yeast, ${ }^{22,23}$ rabbit, ${ }^{24}$ and human $\mathrm{ENO} 2^{25}$ enolases have shown that each polypeptide subunit contains at least two major domains with distinct roles. The $\mathrm{C}$-terminal domain folds into an $\alpha / \beta$ barrel with a typical sequence of $\beta_{2} \alpha_{2}(\beta \alpha) 6$ in secondary structure which forms the active site, ${ }^{24}$ while the $\mathrm{N}$-terminal domain contains a long, flexible loop that folds back onto the active site. ${ }^{26,27}$ ENO catalytic activity has an absolute requirement for two divalent cations $\left(\mathrm{Mg}^{2+}\right)$ binding at distinct sites: a substrate-binding site which induces a conformational change and a chelation site which positions the N-terminal 'flap' over the active site entrance. ${ }^{21-28}$ ENO1-3 catalyze the reversible elimination of water from 2-phosphoglycerate (2-PGA) to form phosphoenolpyruvate, and the two active site magnesium ions apparently facilitate the reaction by activating the $\mathrm{C} 2$ proton of 2-PGA and stabilizing the charged intermediate. ${ }^{28}$

Structures of three human enolase $(E N O)$ genes have been reported, including human ENO1, ${ }^{29}$ ENO2, ${ }^{9}$ and ENO3. ${ }^{30}$ These genes contained 12 exons and showed a high degree of sequence conservation and consistency in the positioning of the introns which suggested a common evolutionary origin for these genes. The $5^{\prime}$-flanking putative gene promoter regions for these genes were also highly conserved and contained a $\mathrm{CpG}$ island in each case. The ENO1 and ENO2 genes lacked canonical TATA and CAAT boxes in the $5^{\prime}$-promoters, which is consistent with these being housekeeping genes, whereas the human ENO3 putative 5'-promoter contained an upstream TATA box. Each of these genes undergoes exon shuffling, generating several isoproteins in each case, ${ }^{31}$ which may perform functions that are distinct from catalyzing the glycolytic reaction. ENO1 isoforms, for example, serve different roles as a hypoxic stress protein, lens crystallin, autoimmune antigen, cell surface plasminogen receptor, and a transcriptional repressor of the Myc proto-oncogene (called myc-binding protein or MBP1)..$^{2-4,32-34}$

This article reports predicted gene structures and amino acid sequences for several vertebrate enolase genes (ENO) and enzymes (ENO) previously not reported, including three closely related enolase family members (ENO1, ENO2, and ENO3) and two other enolase-like genes and proteins (ENO4 and ENO5) that have not been extensively investigated. Predicted secondary and tertiary structures for vertebrate enolases and conserved regulatory regions for mammalian ENO promoters are also described as well as the structural and evolutionary relationships of these genes and enzymes.

\section{Methods}

\section{Vertebrate enolase gene and protein identification}

Basic Local Alignment Search Tool (BLAST) studies were undertaken using Web tools from the National Center for Biotechnology Information (see http://blast.ncbi.nlm.nih. gov/Blast.cgi). ${ }^{35}$ Protein BLAST analyses used human ENO1, ENO2, ENO3, ENO4, and ENO5 amino acid sequences which are previously described (Table 1). Nonredundant protein sequence databases for several vertebrate genomes were examined using the BLASTP algorithm, including human (Homo sapiens), ${ }^{11}$ orangutan (Pongo abelii) (see http://genome.wustl.edu), rhesus monkey (Macaca mulatta), ${ }^{36}$ marmoset (Callithrix jacchus) (see http://genome. ucsc.edu/), cow (Bos taurus), ${ }^{37}$ horse (Equus caballus), ${ }^{38}$ mouse (Mus musculus) ${ }^{39}$ rat (Rattus norvegicus),${ }^{40}$ opossum (Monodelphis domestica) ${ }^{41}$ chicken (Gallus gallus) ${ }^{42}$ frog (Xenopus tropicalis), ${ }^{43}$ zebrafish (Danio rerio), ${ }^{44}$ and nematode (Caenorhabditis elegans) (see http://genome.ucsc.edu/). This procedure produced multiple BLAST 'hits' for each of the protein databases, which were individually examined and retained in FASTA format, and a record was maintained of the sequences for predicted mRNAs and encoded ENO-like proteins. These records were derived from annotated genomic sequences using the gene prediction method: GNOMON and predicted sequences with high similarity scores for human ENO1, ENO2, ENO3, ENO4, and ENO5 (or ENOF1) (see Tables 1 and 2). Predicted ENO-like protein sequences 


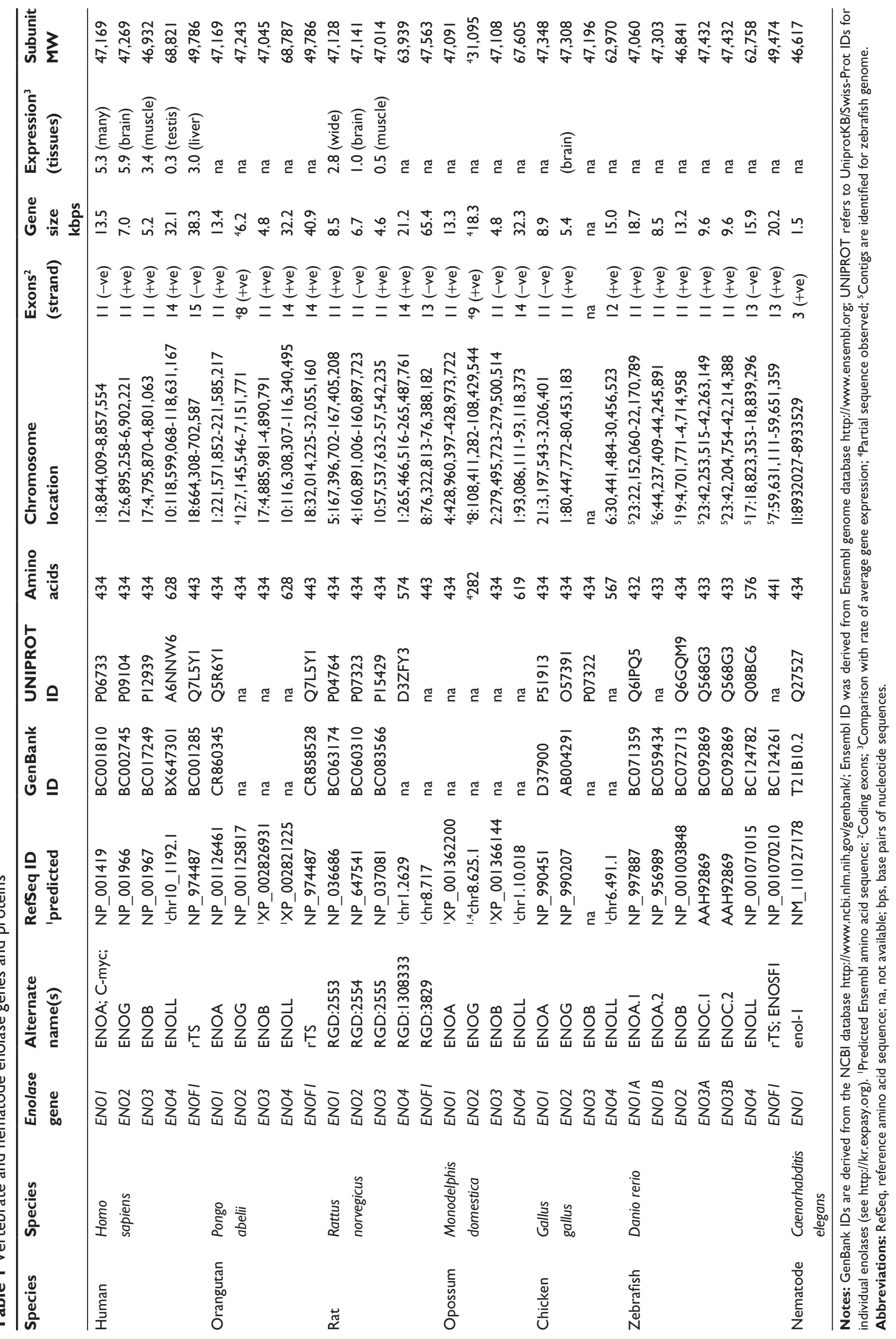




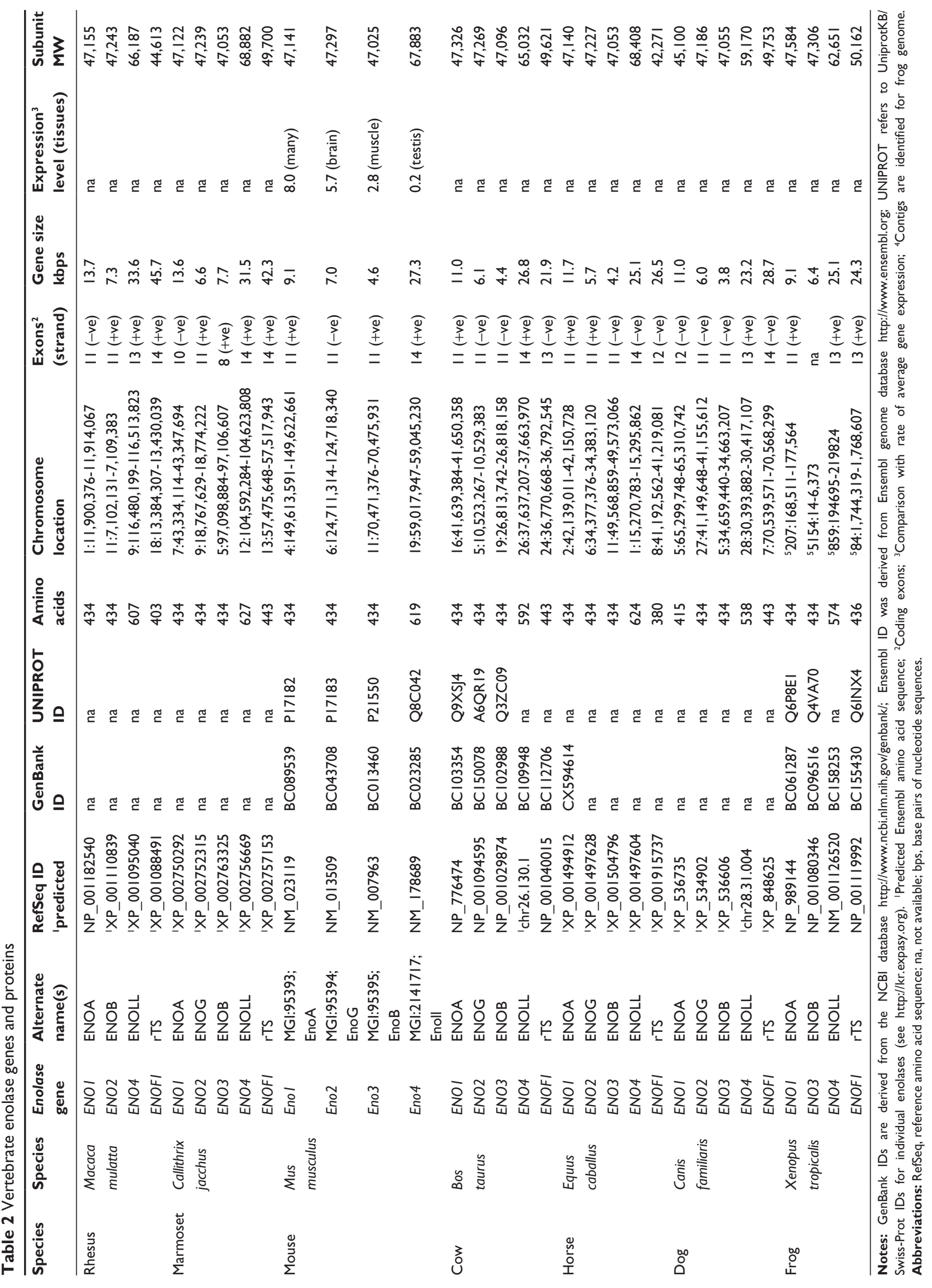


were then subjected to analyses of predicted protein and gene structures.

BLAT analyses were subsequently undertaken for each of the predicted ENO amino acid sequences using the UC Santa Cruz genome browser (see http://genome.ucsc.edu/cgibin/hgBlat $)^{45}$ with the default settings to obtain the predicted locations for each of the vertebrate ENO-like genes, including predicted exon boundary locations and gene sizes for coding exons. Structures for human ENO1, ENO2, ENO3, $E N O 4$, and $E N O 5$ isoforms (splicing variants) were obtained using the AceView Web site to examine predicted gene and protein structures (see http://www.ncbi.nlm.nih.gov/IEB/ Research/Acembly/index.html?human). ${ }^{31}$ The UC Santa Cruz genome browser (http://genome.ucsc.edu) ${ }^{45}$ was used to examine comparative structures for vertebrate and C. elegans enolase genes and for identifying predicted $\mathrm{CpG}$ islands and transcription factor binding sites (TFBS) for human ENO1, ENO2, ENO3, ENO4, and ENO5 genes.

\section{Predicted structures, properties, and alignments of vertebrate enolase-like sequences}

Predicted secondary and tertiary structures for human and other vertebrate ENO-like proteins were obtained using the
PSIPRED v2.5 Web site tools (see http://bioinf.cs.ucl.ac.uk/ psipred/psiform.html) ${ }^{46}$ and SWISS-MODEL Web tools (see http://swissmodel.expasy.org/), respectively. ${ }^{47,48}$ The reported tertiary structure for human $\mathrm{ENO}^{49}$ was used as the reference for the ENO1 tertiary structure, with a modeling range of residues 3-432; the reported structure for human ENO2 served as a reference for human ENO2 and ENO3 (modeling range of 2-431), ${ }^{25,50}$ and the structure for Escherichia coli enolase served as a reference for human ENO4 (modeling range of 67-577). ${ }^{51}$ Alignments of human ENO1-5 sequences were assembled using the ClustalW2 multiple sequence alignment program (see http://www.ebi.ac.uk/Tools/clustalw2/ index.html). ${ }^{52}$

\section{Results and discussion Alignments of human enolase amino acid sequences}

Amino acid sequence alignments for previously reported human (H. sapiens) ENO1 $(\alpha),{ }^{49,53}$ ENO2 $(\gamma),{ }^{8,9,54}$ ENO3 $(\beta),{ }^{24,55}$ ENO4 (also called ENOLL), ${ }^{12}$ and ENO5 (or ENOF1) ${ }^{13}$ sequences are shown in Figure 1 (also see Table 1). The amino acid sequences for the human ENO1, ENO2, and ENO3 subunits contained 434 residues, whereas the predicted human ENO4 and ENO5 sequences (deduced from respective

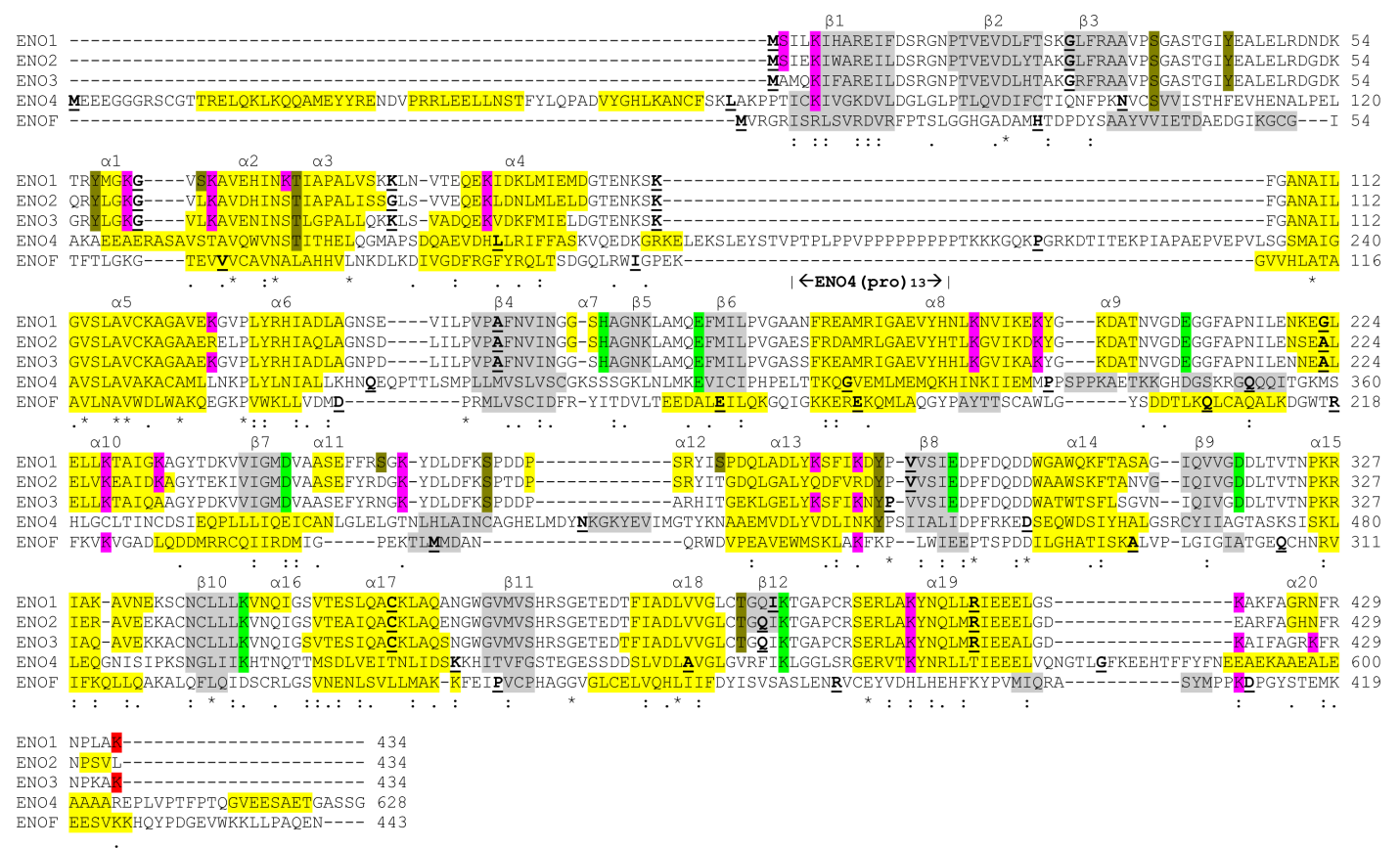

Figure I Amino acid sequence alignments for human ENOI, ENO2, ENO3, ENO4 (ENOLL), and ENO5 (ENOFI) sequences. See Table I for sources of human ENOI, ENO2, ENO3, ENO4, and ENO5 sequences (the latter two are predicted sequences); Symbol * shows identical residues for proteins; :, similar alternate residues; ., dissimilar alternate residues; key enolase active site, $\mathrm{Mg}^{2+}$-binding and substrate-binding residues are in shaded green; predicted or known sites for acetylation are in shaded purple; predicted or known sites for phosphorylation are in shaded khaki; conserved C-terminal lysine residues for human ENOI and ENO3 are in shaded red; $\beta$-sheets ( $\beta$ I- $\beta$ I 2 ) are numbered according to human ENOI sequences ${ }^{49}$ and are in shaded grey; $\alpha$-helices are also numbered according to the ENOIstructure ${ }^{47}$ and are in shaded yellow; bold underlined font shows residues corresponding to known or predicted exon start sites; exon numbers refer to human ENOI gene coding exons. 


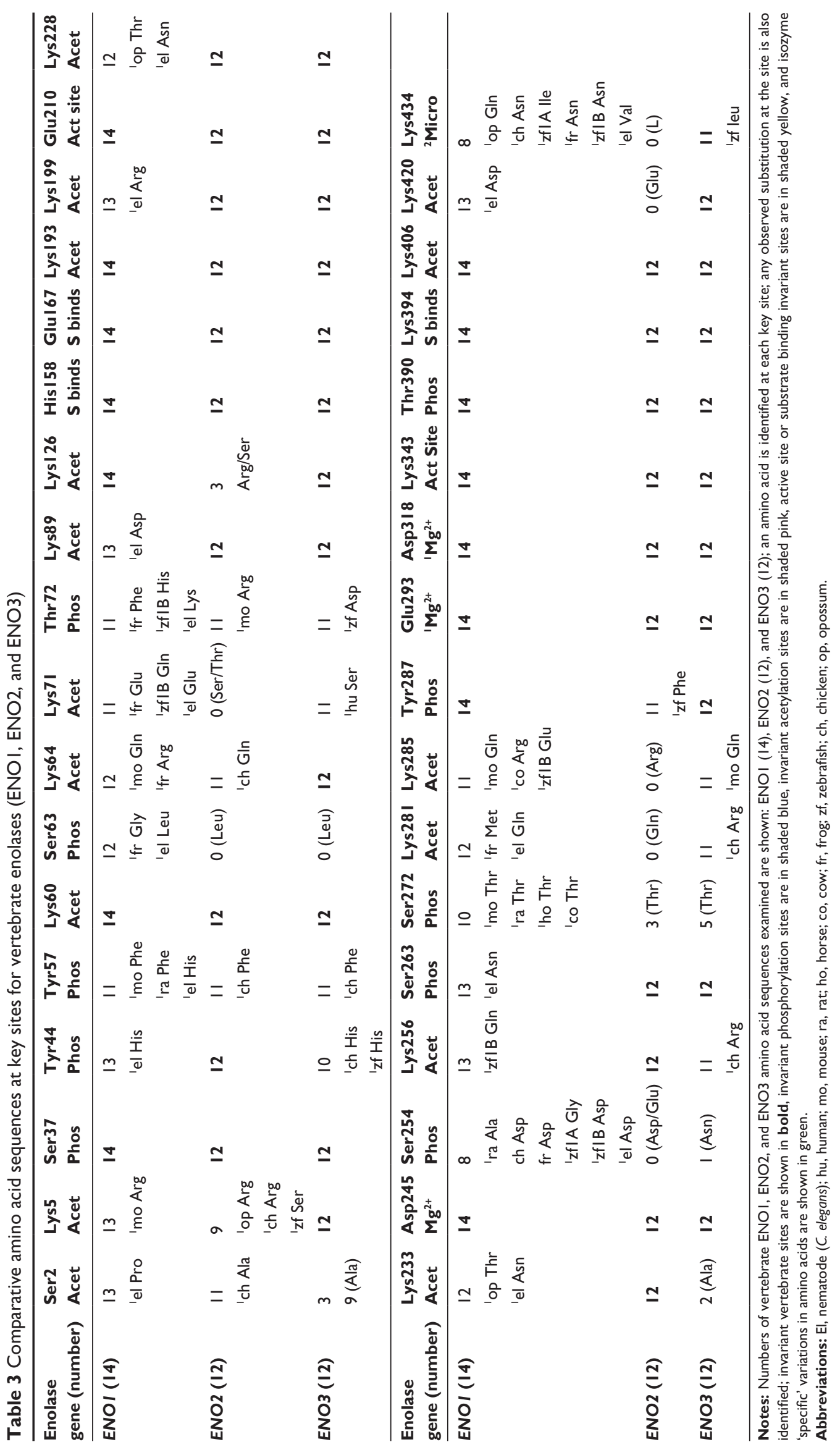


nucleotide sequences in each case) contained 628 and 443 amino acids, respectively (Figure 1).

Previous biochemical and genetic analyses of human and mouse ENO1, ${ }^{49,56,57}$ ENO2, ${ }^{8,9,58}$ and $\mathrm{ENO}^{34,59}$ have enabled predictions of key residues for human ENO1, ENO2, and ENO3. These included active site residues: Glu210 (proton donor), Lys343 (proton acceptor), His158, Glu167, Glu293, Asp318, and Lys394 (substrate-binding sites); $\mathrm{Mg}^{2+}$ chelating sites: Asp245, Glu293, and Asp318 (required for catalysis and stabilizing the dimer); C-terminal Lys434 for ENO1 and ENO3 (required for the binding to neuronal and skeletal muscle plasma membranes, respectively); acetylated residues: Ser2 and Lys residues 60, 64, 71 (ENO1), 89, 126 (ENO1 and ENO3), 193, 199, 228, 233 (ENO1 and ENO2), 256, 281, and 285 (ENO1 and ENO3), 406 and 420 (ENO1 and ENO3); and phosphorylated residues Ser37, 63, and 263 (ENO1) and 272; Tyr44, 57, and 287; and Thr72 and 390 (residues in bold are shared among human ENO1, ENO2, and ENO3 sequences). Given the potential roles of acetylated and phosphorylated ENO sites in regulating cellular metabolism, ${ }^{56,57}$ the existence of conserved and isozyme-specific sites are of particular significance for these enzymes.

Table 3 compares the amino acid residues localized in each of these key sites for ENO1, ENO2, and ENO3 from 13 vertebrate species and for ENO1 obtained for the nematode, C. elegans. In addition to the active site and substrate-binding and $\mathrm{Mg}^{2+}$-chelating sites, several previously described acetylation and phosphorylation sites were also strictly conserved among the ENO sequences examined. These included phosphorylation sites Ser37, Ser263, and Thr390; and lysine acetylation sites 60, 89, 193, 199, and 406. In contrast, there are a number of predicted 'isozyme'specific translationally modified sites, including two ENO1 phosphorylated residues: Ser63 (ENO1)/Leu63 (ENO2 and ENO3); Ser254 (ENO1)/Asp or Glu (ENO2) and Asn (ENO3); and four ENO1 acetylated residues: Lys71 (ENO1 and ENO3)/Ser or Thr71 (ENO2); Lys281 (ENO1 and ENO3)/Gln281 (ENO2); Lys285 (ENO1 and ENO3)/Arg285 (ENO2); and Lys420 (ENO1 and ENO3)/Glu420 (ENO2). In addition, the C-terminal 434Lys, previously shown to play a microlocalization role for ENO1 in neuronal cells ${ }^{17,18}$ and for ENO3 in binding the $\beta \beta$-isozyme to muscle filaments, ${ }^{7}$ is predominantly conserved among the vertebrate sequences examined, but has been substituted with Leu434 for the 12 vertebrate ENO2 sequences. With the exception of the differences for the $\mathrm{C}$-terminal residue, the significance of these isozyme-specific changes in sequences remains to be determined.
Alignments of vertebrate ENO1, ENO2, and ENO3 and nematode ENO1 amino acid sequences examined showed between $69 \%$ and $97 \%$ identities, suggesting that these are products of one gene family, which is highly conserved during vertebrate and invertebrate evolution (Table 4). In addition, sequences of multiple zebrafish (D. rerio) ENO1 (designated as ENO1A and ENO1B) and ENO3 (ENO3A and ENO3B) also showed similar or identical sequences $(88 \%$ and $100 \%$, respectively). Comparisons of the vertebrate ENO1-3 sequences with the predicted ENO4 (also called ENOLL) and ENO5 (also called ENOF1), however, showed large differences in sequence identity, with only $\sim 20 \%$ identical sequences for the ENO1-3 proteins with ENO4 and $<10 \%$ with ENO5, suggesting that the latter are members of distinct $E N O$ gene families.

\section{Secondary and tertiary structures for vertebrate enolases}

Predicted secondary structures for human ENO4 and ENO5 sequences were compared with those previously reported for human ENO1, ${ }^{49,57}$ ENO2, ${ }^{23,25}$ and ENO3 ${ }^{55}$ (Figure 1). $\alpha$-Helix and $\beta$-sheet structures for these sequences were numbered as for those described for human ENO1. ${ }^{49}$ The predicted human ENO4 secondary structure ${ }^{51}$ was similar to those for human ENO1-3 sequences, although a number of additional structures were observed, including three $\mathrm{N}$-terminal $\alpha$-helices; a poly-proline ENO4 sequence (residues 177-233); two $\beta$-sheets (designated as ENO4 $\beta 5$ and $\beta 6$ ) (residues 394-415); and an extended C-terminal sequence (residues 605-628) containing an $\alpha$-helix. The predicted ENO5 secondary structure also exhibited similarities with the human ENO1-3 secondary structures which were previously reported ${ }^{25,49,55}$ although major differences were apparent, particularly in the extended C-terminal region. Given that the ENO5 (also called ENOF1) gene has a proposed role in encoding an antisense transcript for $T S^{13}$ and in regulating the $T S$ locus ${ }^{14}$ it is likely that ENOF1 does not function in catalyzing the glycolytic enolase reaction. This is supported by examining the predicted human ENO5 amino acid sequence (Figure 1) for which several key ENO residues have been substituted, including the active site residues and conserved acetylated and phosphorylated residues for ENO1-3.

Figure 2 compares previously reported structures for human $\mathrm{ENO} 1,{ }^{49} \mathrm{ENO} 2,{ }^{23,25}$ and $\mathrm{ENO} 3{ }^{55}$ protein sequences with a predicted tertiary structure for the human ENO4 (or ENOLL) subunit (based on the reported tertiary structure for E. coli enolase ${ }^{51}$ ). Two major differences were observed 


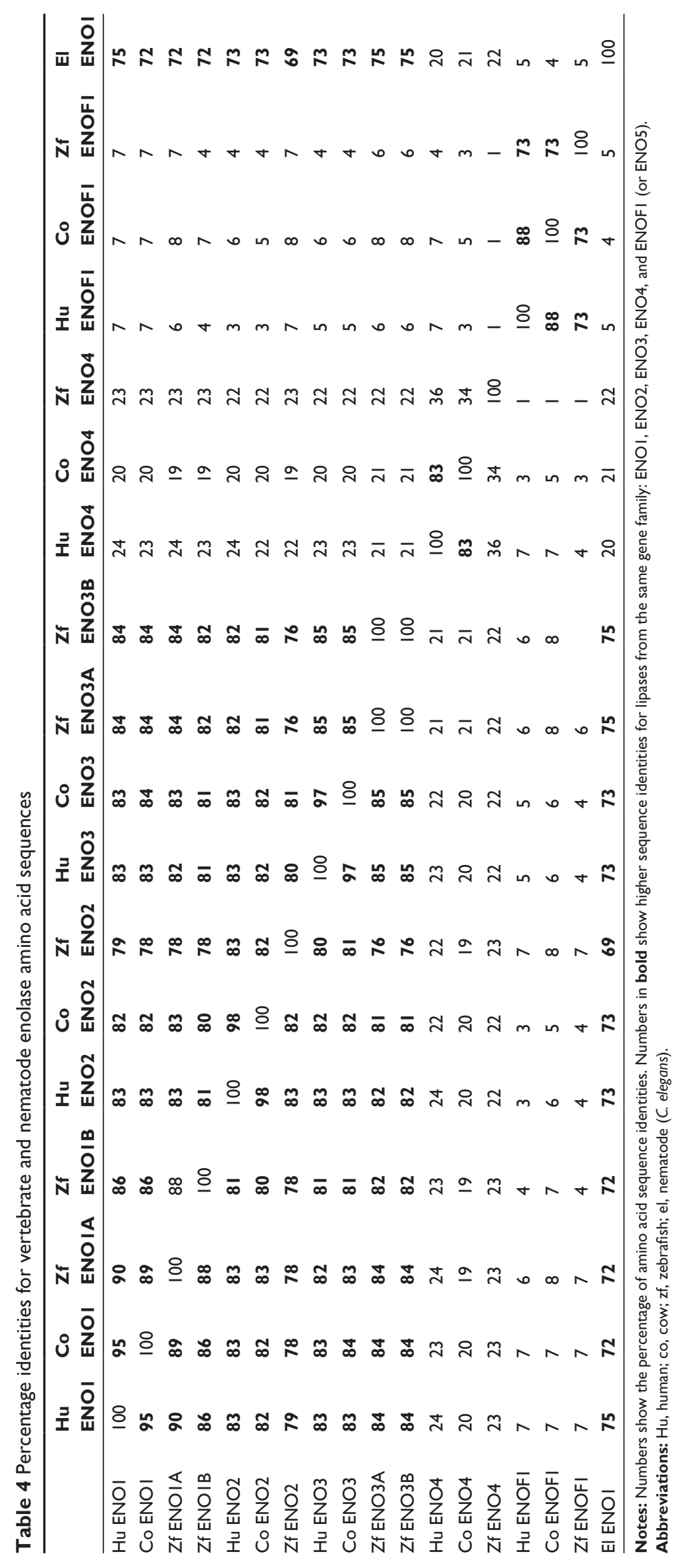


for the ENO4 tertiary structure: an extended chain region corresponding to a poly-proline amino acid segment (ENO4 residues 177-232) not present in the human ENO1-3 structures and two additional $\beta$-sheet segments (human ENO4 residues 334-354 and 394-415) (also see Figure 1). The rest of the predicted ENO4 structure was similar to those previously described for human ENO1-3 subunits, although the homology model for ENO4 did not include the extended N-terminal and C-terminal regions. Although ENO4 displays a similar structure to the human ENO1-3 active site zone (see Figure 2), the amino acid alignments results (Figure 1) show that ENO4 lacks active site and other key residues and would not be expected to function in catalyzing the glycolytic reaction and an alternate role should be considered.

\section{Gene locations and exonic structures for vertebrate ENO genes}

Tables 1 and 2 summarize the predicted locations for vertebrate and nematode (C. elegans) enolase-like (ENO) genes based upon BLAT interrogations of genomes using the reported sequences for human ENO1 $(\alpha),{ }^{49,53} \operatorname{ENO} 2(\gamma),{ }^{8,9}$ ENO3 $(\beta),{ }^{55}$ ENO4 (also called ENOLL), ${ }^{12}$ and ENO5 (or ENOF1) ${ }^{13}$ and the predicted sequences for other vertebrate ENO-like proteins using the UC Santa Cruz genome browser. ${ }^{45}$ The mammalian $E N O$-like genes were transcribed on either strand, depending on the ENO gene or genome examined. Figure 1 summarizes the coding exon start sites for human ENO1, ENO2, and ENO3 genes, showing 11 coding exons in identical or similar positions which are consistent with previous reports. ${ }^{9,53,54}$ In contrast, the human ENO4 gene contained 13 coding exons, including two additional exons encoding an extended $\mathrm{N}$-terminal sequence and a poly-proline segment not observed in the human ENO1-3 and ENOF1 sequences. In addition, the ENO4 coding exon start sites were in distinct positions to those reported for human ENO1, ENO2, and ENO3 genes. Comparisons of predicted $E N O 4$ vertebrate gene structures showed that each contained predominantly 14 coding exons, whereas dog, frog, and zebrafish ENO4 genes contained 13 coding exons. Genomic analyses of the human ENO5 gene showed that 15 predicted coding exons were present, which corresponded to different locations to those previously reported for human $E N O 1, E N O 2$, and $E N O 3^{9,53,54}$ and described here for human ENO4 (Figure 1). Comparative analyses of vertebrate ENO5 genes showed that the number of coding exons varied with the species examined, from 12 for the horse ENO5 (or ENOF1) gene to 15 for the human ENO5 gene.
Figure 3 shows the predicted structures of $\mathrm{mRNAs}$ for human ENO1, ENO2, ENO3, ENO4, and ENO5 transcripts for the major isoform in each case. ${ }^{31}$ These human mRNA transcripts varied in length from 5.2 to $13.5 \mathrm{~kb}$ for the ENO1-3 genes and up to 32 and $38 \mathrm{~kb}$ for human ENO4 and ENO5 genes, respectively. The human ENO1-3 gene transcripts contained extended $5^{\prime}$-untranslated (UTR) and 3 '-UTR regions, with the latter also observed for the ENO4 gene and the former for the human ENO5 gene. Human ENO1 and ENO5 transcripts were encoded on the negative strand, whereas human ENO2, ENO3, and ENO4 transcripts were transcribed on the positive strand (Table 1). In each case for these human ENO genes, a $\mathrm{CpG}$ island was observed within the $5^{\prime}$-regulatory promoter regions for these genes. CpG islands are typically observed within the promoters of housekeeping genes and may enhance a high level of expression for these genes. ${ }^{60}$ The levels of expression for these human ENO genes have been compared with the average level of gene expression observed in the human genome ${ }^{31}$ (see Table 1). Four of these genes exhibited higher levels of expression (ENO1 (x5.3), ENO2 (x5.9), ENO3 (x3.4), and ENO5 (x3.0)), whereas human ENO4 exhibited a lowerthan-average level of expression (x0.3). The higher levels of ENO1-3 expression were also observed for the mouse and rat genes (see Tables 1 and 2).

In addition to the $\mathrm{CpG}$ islands observed for the human ENO gene promoters, these sequences also contained 78 predicted TFBS including within the $5^{\prime}$-upstream promoter region (20 sites), 5'-UTR promoter region ( 7 sites), and intron 1 (seven sites), which are usually associated with regulating gene transcription (Table 5). Several of these sites have been shown to play significant roles in regulating ENO gene expression. For example, the HIF1 site (hypoxia-inducible factor located in the $5^{\prime}$-UTR region of ENO1) activates genes encoding proteins that mediate responses to hypoxia, in association with coactivator proteins, such as the CREB-binding protein, ${ }^{2,61}$ which also has a binding site in this region. An alternate translated form of ENO1 (named MBP1), which is predominantly located in the nucleus, has been characterized as a c-Myc promoter-binding protein that negatively controls transcription of this proto-oncogene ${ }^{32}$ which identifies ENO1 as a potential tumor suppressor. Sousa and coworkers ${ }^{62}$ have also shown that interferons induce $E N O 1$ expression in target cells by activating mitogen-activated protein kinases and the transcription factor (CREB). The $5^{\prime}$-noncoding exon for $E N O 3$ contains many predicted motifs for transcriptional regulation, including $\mathrm{Sp} 1$, activator protein 1 and 2, CCAAT box transcription factor/nuclear factor 1, and 


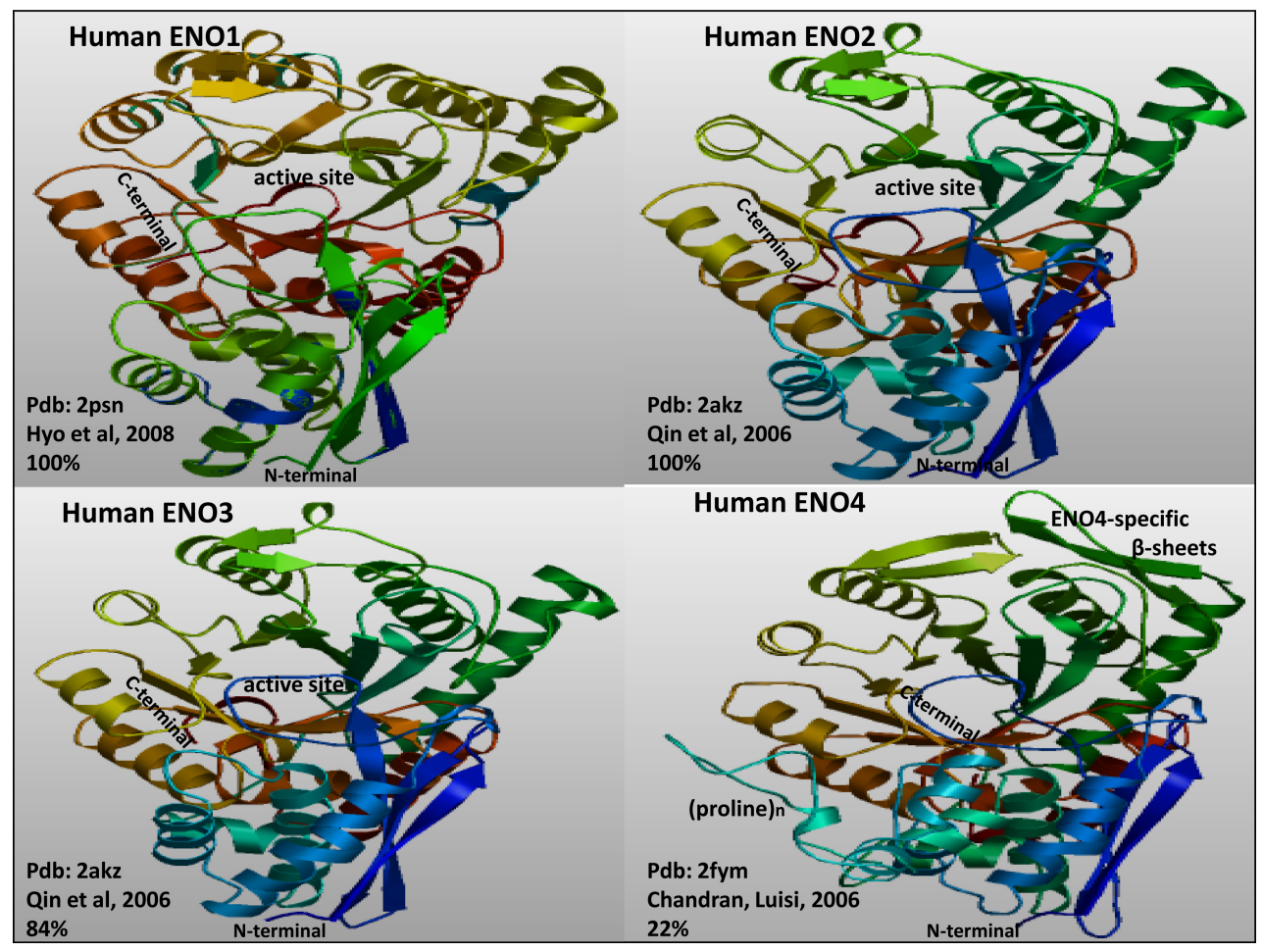

Figure 2 Known or predicted tertiary structures for human ENOI, ENO2, ENO3, and ENO4 (ENOLL). Tertiary structures were obtained using SWISS-MODEL methods; the rainbow color code describes the known tertiary structures from the $\mathrm{N}$ - (blue) to C-termini (red color) for human ENOI, ${ }^{99} \mathrm{ENO}^{25}$ and ENO3,55 and the predicted structure for human ENO4 (ENO4 (ENOLL) structure based on E. coli enolase $\left.\mathrm{s}^{51}\right)$; arrows indicate directions for $\beta$-sheets; known or predicted active site, $\mathrm{N}$-terminal and C-terminal regions are shown, as are predicted structures and locations for ENO4 $\beta 5$ and $\beta 6$ sheets and a poly-proline ((proline)n) sequence.

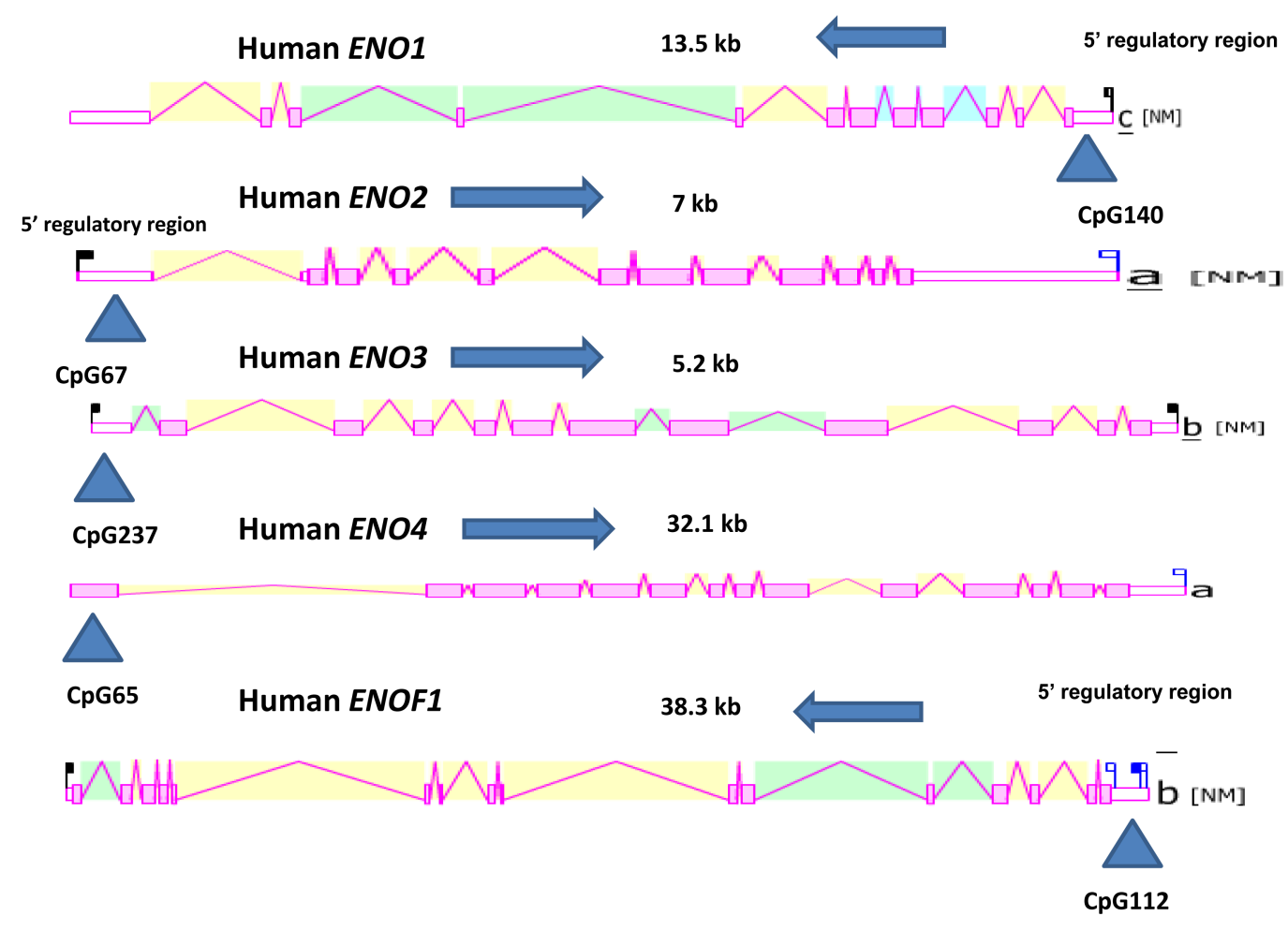

Figure 3 Gene structures and major splicing variant for human ENOI, ENO2, ENO3, ENO4, and ENO5 (ENOFI) gene transcripts. Derived from AceView, ${ }^{31}$ mature isoform variants (a) are shown with capped $5^{\prime}$ - and $3^{\prime}$-ends for the predicted mRNA sequences, NM refers to the NCBI reference sequence, exons are in shaded pink, untranslated $5^{\prime}$ - and $3^{\prime}$-sequences are in open pink, introns are represented as pink lines joining exons, the directions for transcription are shown as $5^{\prime} \rightarrow 3^{\prime}$, sizes of mRNA sequences are shown in kilobases (kb), $C_{P G}$ islands are identified and numbered. (a), (b) and (c) refer to the major isoforms of enolase genes. 
cyclic AMP, and for muscle-specific ENO3 gene regulation, a CC(A + T-rich) 6GG box, M-CAT-box CAATCCT, and two myocyte-specific enhancer-binding factor 1 boxes. ${ }^{30,63}$ Muscle-specific ENO3 gene enhancers are also located within the first intron that bind myocyte-specific enhancer factor 2 proteins and G-rich-box binding factors. ${ }^{64}$ A TFBS (AHR1) and a $\mathrm{CpG}$ island for regulating ENO4 gene expression were also identified in the $5^{\prime}$-UTR region for ENO4, which may suggest a role for ENO4 (or ENOLL) in aryl hydrocarbon ligand binding or metabolism.

\section{Comparative tissue expression of mouse enolase genes and differential functions for vertebrate enolases}

Figure 4 presents 'heat maps' showing comparative gene expression for various mouse tissues obtained from GNF Expression Atlas Data using GNF1M (mouse) chips ${ }^{65}$ (see http://genome.ucsc.edu; http://biogps.gnf.org). These data supported differential tissue expression for mouse enolase genes: Enol showing highest levels in embryonic tissues, kidney, and brown adipose tissue; Eno2 with highest expression levels in neural tissues; Eno3 with highest levels of expression in skeletal muscle, heart, brown fat, bone, and prostate; and Eno5 (or Enof1) showing a broad tissue expression profile. This is consistent with previous reports for these genes. ${ }^{59,66,67}$ There were no 'heat map' results available for the mouse Eno4 gene. Overall, however, mouse and human ENO1, ENO2, ENO3, and ENO5 (or ENOF1) tissue gene expression levels were $>3$ times higher than the average level of gene expression which supports the key role played by these proteins and enzymes in glycolysis and in various multifunctional roles in the body (for ENO1-3) ) $^{23,49}$ and in regulating TS activity (ENOF1). ${ }^{13,14}$ In contrast, the average human and mouse ENO4 gene expression was below the average $(\times 0.2-0.3)$ with highest levels observed in testis in each case (Tables 1 and 2).

Enolase genes and proteins are multifunctional with the three major genes (ENO1, ENO2, and ENO3) encoding $\alpha-, \gamma-$, and $\beta$-subunits which form dimeric isozymes, performing a primary role in glycolysis, catalyzing 2-phospho-D-glycerate hydro-lyase activity. ${ }^{1}$ These isozymes are also subject to differential localization within the cell. The $\alpha$-subunit contains a C-terminal lysine residue, which facilitates binding with plasminogen in the neuronal plasma membrane and promotes its activation, ${ }^{8,49}$ while the $\beta$-subunit $\mathrm{C}$-terminal lysine facilitates binding to troponin in the Z-line of striated muscle fibers. ${ }^{68,69}$ A reported association of a genetic deficiency for the enolase $\beta$-subunit with muscle weakness supports a role for this isozyme in localized adenosine triphosphate production within muscle fibers. ${ }^{70}$ The physiological importance of enolase subcellular localization has also been demonstrated in studies of flagellar motility and energy production in Chlamydomonas reinhardtii. ${ }^{71}$ ENO1 $(\alpha \alpha)$ also plays a role in hypoxia tolerance, ${ }^{2}$ tumor suppression, ${ }^{3}$ cell surface plasminogen binding, ${ }^{4}$ or acting as a lens tau-crystallin. ${ }^{5}$ A differentially translated isoform of ENO $\alpha \alpha$ (called MBP-1) also binds to the c-Myc promoter and acts as a transcriptional repressor and DNA-binding protein and is a potential candidate as a tumor suppressor. ${ }^{6,32}$

In contrast to the multifunctional roles in carbohydrate metabolism and other processes for $\alpha$-, $\beta$-, and $\gamma$-subunits containing enolases, ENO5 (ENOF1 or ENOSF1) was originally identified as encoding an antisense transcript to the $T S$ gene ${ }^{13}$ with a proposed role in regulating the $T S$ locus by the synthesis of signaling molecules involved in the downregulation of TS. ${ }^{14}$ ENO4 (or ENOLL) has also been reported as an enolase-like gene ${ }^{12}$ but has only been described at the transcript level as yet. The lack of active site residues for this 'predicted protein' is suggestive of another function similar to that of catalyzing enolase activity, either as a protein (ENO4 or ENOLL) or as a transcript. The predicted 3-D structure for ENO $4^{51}$ shows significant similarities with the $\alpha-, \beta$-, and $\gamma$-subunits, although with two additional $\beta$-sheets which may overlay the active site and an extended poly-proline chain, which may suggest another function to that of catalyzing enolase activity (Figure 2). It is relevant to note that the homology modeling method used to derive a predicted 3-D structure for human ENO4 was based on E. coli enolase ${ }^{51}$ rather than on a mammalian $\alpha-, \beta$-, and $\gamma$-containing subunit structure. E. coli enolase also plays a role within a multienzyme complex called the RNA degradosome ${ }^{72}$ which may suggest a similar role for human ENO4. The location of a TFBS (AHR1) within the ENO4 promoter region also suggests a role for ENO4 (or ENOLL) in aryl hydrocarbon ligand binding or metabolism.

\section{Phylogeny and divergence of enolase sequences}

A phylogenetic tree (Figure 5) was calculated by the progressive alignment of 39 vertebrate ENO1, ENO2, and ENO3 amino acid sequences with the nematode (C. elegans) enolase sequence serving as the 'root' for the tree (see Tables 1 and 2). The phylogram showed clustering of the ENO sequences into three groups which were consistent with their evolutionary relatedness, as well as groups for ENO1, ENO2, and ENO3, 


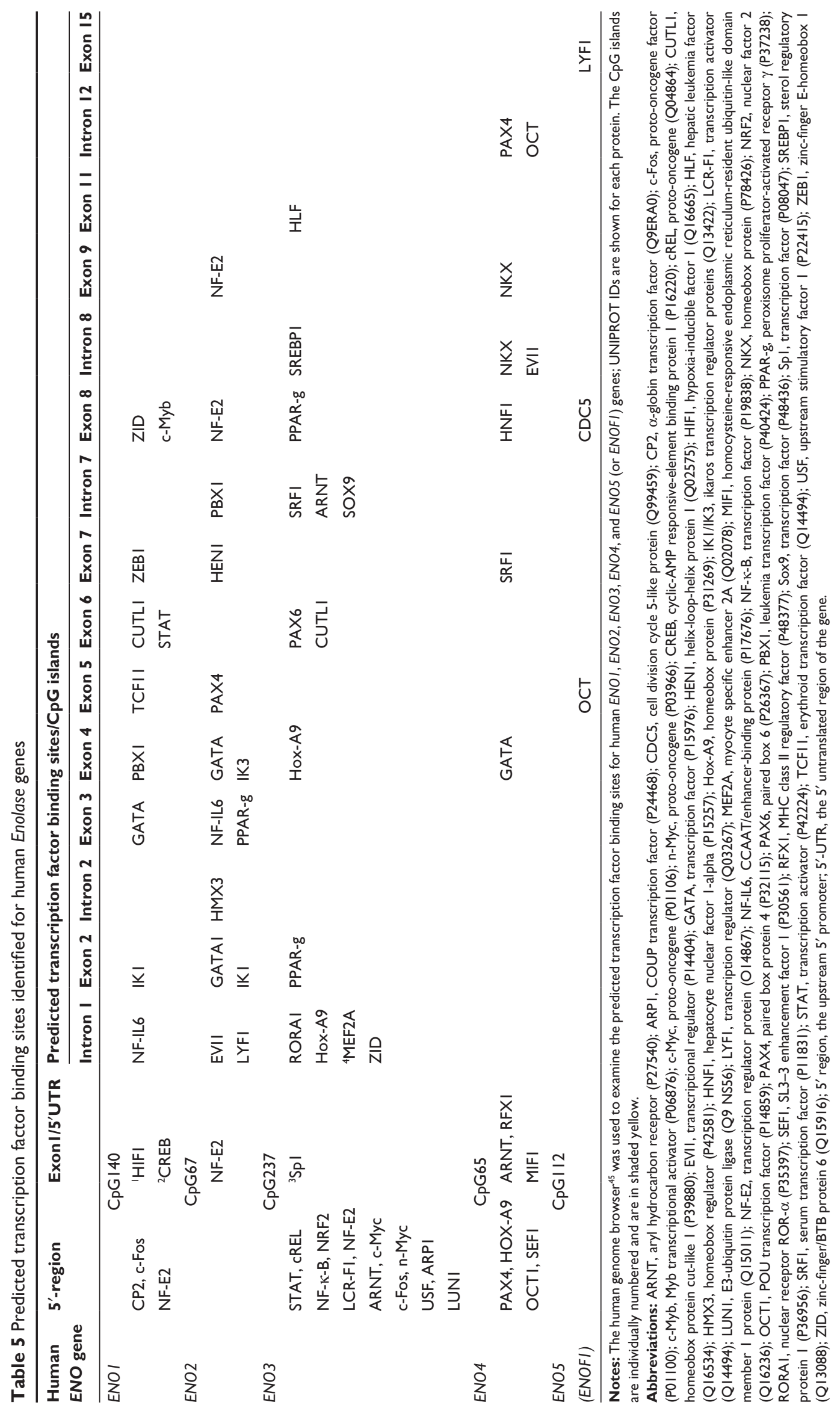




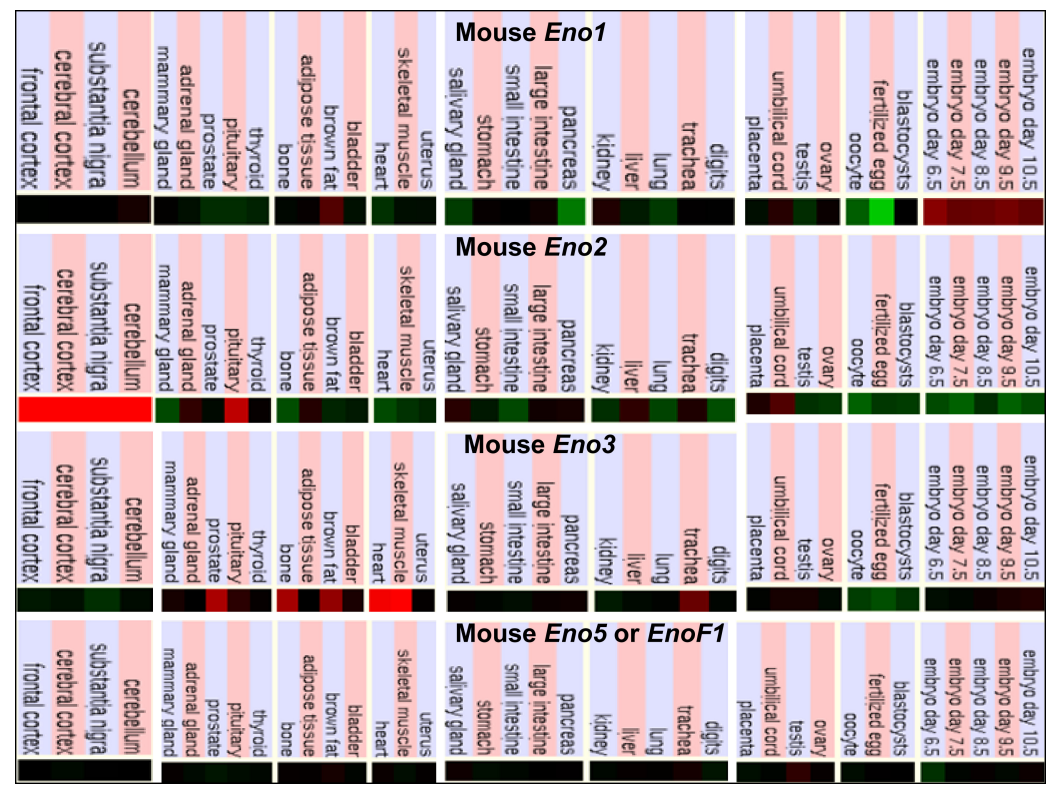

Figure 4 Comparative tissue expression for mouse enolase genes (Enol, Eno2, Eno3, and Enofl). Expression 'heat maps' (GNF Expression Atlas 2 data) ${ }^{65}$ were examined for comparative gene expression levels among mouse tissues for Enol, Eno2, Eno3, and Eno5 (or Enofl) genes showing high (red), intermediate (black), and low (green) expression levels, derived from mouse genome browser. ${ }^{45}$

and which were significantly different from each other (with bootstrap values of 91-100). It is apparent from this study of vertebrate ENO1, ENO2, and ENO3 genes and proteins that these are ancient proteins for which a proposed common ancestor for these genes may have predated the appearance of fish $>500$ million years ago ${ }^{73}$ Tracy and Hedges ${ }^{20}$ have examined this timing event further and have concluded that the ENO1 and ENO3 genes appeared first in actinopterygian, sarcopterygian, and chondrichthyan fishes and that the third gene duplication event generating ENO2 occurred subsequently to the divergence of living agnathans (jawless fish, eg, lamprey) ( $\sim 550$ million years ago). Liang and coworkers ${ }^{14}$ have also conducted a comprehensive phylogenetic analysis of ENO5 (also called ENOF1 and rTS) protein and showed that it has an extended distribution profile and exists in some groups of eubacteria, two fungal lineages, and most animal species from insects to mammals, demonstrating that $E N O F 1$ (ENO5) is a very ancient gene in biological evolution. There are no reports available on the phylogeny of the vertebrate ENO4 (or ENOLL) protein, although this present study shows that the gene is present among all vertebrate genomes examined.

\section{Conclusion}

These results demonstrate that vertebrate enolase (ENO) genes and encoded enzymes (ENO) comprise at least three distinct forms of enolases: 1) ENO1, ENO2, and ENO3;
2) ENO4; and 3) ENO5 (or ENOF1). The first group is further subdivided into three families corresponding to ENO1, ENO2, and ENO3 genes, observed for each of the vertebrate genomes examined and previously reported for many mammals and chicken. ${ }^{8-11}$ ENO1, ENO2, and ENO3 enzymes not only have a primary enzymatic role in catalyzing the 2-phospho-D-glycerate hydro-lyase activity in glycolysis ${ }^{1}$ but also perform a number of other functions. For ENO1, these include a role in hypoxia tolerance, ${ }^{2}$ tumor suppression, ${ }^{3}$ cell surface plasminogen binding, ${ }^{4}$ and acting as a lens tau-crystallin, ${ }^{5}$ and for an isoform of ENO1 (called MBP-1), the functions include binding to the c-Myc promoter and serving as a potential tumor suppressor. ${ }^{6,30}$ In contrast, vertebrate ENO4 genes have only been described at the transcript level, ${ }^{12}$ and little is known concerning the potential role(s) of the vertebrate ENO4 gene, its transcript, or encoded protein, although the gene is present throughout vertebrate evolution. ENO5 (ENOF1 or ENOSF 1) was originally identified as an antisense transcript to the TS gene, and a mitochondrial protein (ENOF1) has been reported which may play a role in regulating the TS locus. ${ }^{13,14}$

ENO1, ENO2, and ENO3 were each encoded by single genes among most vertebrate genomes examined, with the exception of the zebrafish genome, which contained two ENO1-like and ENO3-like genes. These genes are highly but differentially expressed in human and mouse tissues, 


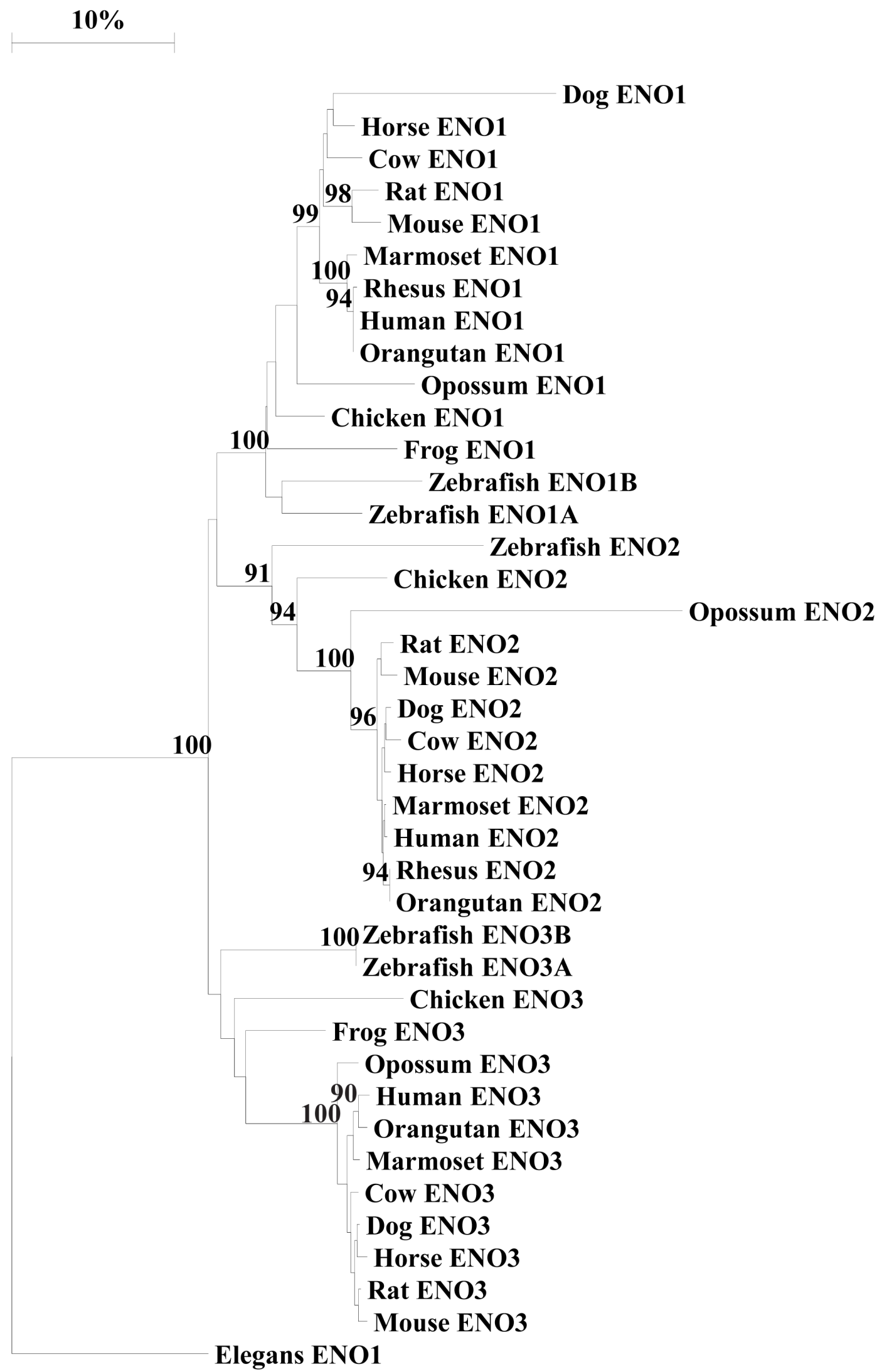

Figure 5 Phylogenetic tree of vertebrate enolase (ENOI, ENO2, and ENO3) with nematode enolase amino acid sequences. The tree is labeled with the enolase name and the name of the animal and is 'rooted' with the nematode enolase sequence (C. elegans). Note the three major clusters corresponding to the ENOI, ENO2, and ENO3 gene families. A genetic distance scale is shown (\% amino acid substitutions). The number of times a clade (sequences common to a node or branch) occurred in the bootstrap replicates are shown. Only replicate values of 90 or more which are highly significant are shown with 100 bootstrap replicates performed in each case. 
with $E N O 1$ expression predominating in embryonic tissues; ENO2 in neural tissues; and ENO3 in skeletal and heart muscles, ${ }^{59,64-66}$ and usually contained 11 coding exons. Predicted structures for human ENO4 ${ }^{51}$ and human ENO5 (Figure 1) proteins showed similarities with human ENO1, ${ }^{49}$ ENO2, ${ }^{23,25}$ and ENO3. ${ }^{55}$ Human ENO4, however, exhibited at least four distinct structures, including an extended $\mathrm{N}$-terminal sequence containing three predicted $\alpha$-helices, two additional $\beta$-sheets, a poly-proline chain, and an extended C-terminal region with an additional predicted $\alpha$-helix. Comparisons of ENO1, ENO2, and ENO3 amino acid sequences from vertebrates representative of mammals, birds, amphibians, and bony fish demonstrated that these are highly conserved proteins during evolution, not only for active site residues, but also for those involved in posttranslational changes, such as acetylation and phosphorylation, and for the C-terminal lysine residue for ENO1 and ENO3 sequences, which participate in localizing the enzyme within cell macromolecular structures. ${ }^{7,68,69}$ Vertebrate ENO1, ENO2, and ENO3 sequences shared 78\%-98\% identities, but only 19\%-24\% with ENO4 and $>10 \%$ predicted sequence identities with vertebrate ENOF1. Sequence alignments, key amino acid residues, and conserved predicted secondary and tertiary structures were examined, including active site residues (absent in ENO4 and ENOF1) and sites for $\mathrm{Mg}^{2+}$ and plasminogen binding and for acetylation and phosphorylation. Mutation studies of yeast enolase have served as useful models for examining specific roles of individual residues and likely impacts of genetic deficiencies for human enolases. ${ }^{74}$

Potential TFBS and $\mathrm{CpG}$ islands for regulating ENO gene expression were identified using bioinformatic techniques. Human ENO1, ENO2, ENO3, and ENOF1 genes each contained $\mathrm{CpG}$ islands in the gene promoter regions consistent with the higher-than-average levels of ENO gene expression observed. Human ENO3 and ENO1 gene promoters also contained a diverse range of TFBS. The ENO4 gene promoter contained a $\mathrm{CpG}$ island and several TFBS, including AHR1 in the 5'-UTR region, which may suggest a role for ENO4 in aryl hydrocarbon ligand binding or metabolism. Phylogeny studies of vertebrate ENO1, ENO2, and $E N O 3$ genes and enzymes suggested that they originated in a vertebrate ancestor from gene duplication events of an ancestral ENO1-like gene $>500$ million years ago, which is consistent with an earlier study ${ }^{20}$ but is in contrast to the ENOF 1 gene which has a much wider biological distribution, including some eubacteria, fungi, as well as invertebrate and vertebrate animals. ${ }^{14}$

\section{Acknowledgments}

The expert assistance and advice of Dr Laura Cox of the Southwest Foundation for Biomedical Research, San Antonio, TX, USA and Dr Bharet Patel of Griffith University, Australia, are gratefully acknowledged.

\section{Disclosure}

The author reports no conflicts of interest in this work.

\section{References}

1. Rider CC, Taylor CB. Enolase isoenzymes in rat tissues. Electrophoretic, chromatographic, immunological and kinetic properties. Biochim Biophys Acta. 1974;365(1):285-300.

2. Semenza GL, Jiang BH, Leung SW, et al. Hypoxia response elements in the aldolase A, enolase 1, and lactate dehydrogenase A gene promoters contain essential binding sites for hypoxia-inducible factor 1. J Biol Chem. 1996;271(51):32529-32537.

3. He P, Naka T, Serada S, et al. Proteomics-based identification of alpha-enolase as a tumor antigen in non-small lung cancer. Cancer Sci. 2007;98(8):1234-1240.

4. Wygrecka M, Marsh LM, Morty RE, et al. Enolase-1 promotes plasminogen-mediated recruitment of monocytes to the acutely inflamed lung. Blood. 2009;113(22):5588-5598.

5. Kim RY, Lietman T, Piatigorsky J, Wistow GJ. Structure and expression of the duck alpha-enolase/tau-crystallin-encoding gene. Gene. 1991;103(2):193-200.

6. Wang W, Wang L, Endoh A, Hummelke G, Hawks CL, Hornsby PJ. Identification of alpha-enolase as a nuclear DNA-binding protein in the zona fasciculata but not the zona reticularis of the human adrenal cortex. J Endocrinol. 2005;184(1):85-94.

7. Keller A, Peltzer J, Carpentier G, et al. Interactions of enolase isoforms with tubulin and microtubules during myogenesis. Biochim Biophys Acta. 2007;1770(6):919-926.

8. McAleese SM, Dunbar B, Fothergill JE, Hinks LJ, Day IN. Complete amino acid sequence of the neurone-specific gamma isozyme of enolase (NSE) from human brain and comparison with the non-neuronal alpha form (NNE). Eur J Biochem. 1988;178(2): 413-417.

9. Oliva D, Barba G, Barbieri G, Giallongo A, Feo S. Cloning, expression and sequence homologies of cDNA for human gamma enolase. Gene. 1989;79(2):355-360.

10. Feo S, Oliva D, Barbieri G, Xu WM, Fried M, Giallongo A. The gene for the muscle-specific enolase is on the short arm of human chromosome 17. Genomics. 1990;6(1):192-194.

11. Lander ES, Linton LM, Birren B, et al; International Human Genome Sequencing Consortium. Initial sequencing and analysis of the human genome. Nature. 2001;409(6822):860-921.

12. Deloukas P, Earthrowl ME, Grafham DV, et al. The DNA sequence and comparative analysis of human chromosome 10. Nature. 2004;429(6990):375-381.

13. Dolnick BJ. Cloning and characterization of a naturally occurring antisense RNA to human thymidylate synthase mRNA. Nucleic Acids Res. 1993;21(8):1747-1752.

14. Liang P, Nair JR, Song L, McGuire JJ, Dolnick BJ. Comparative genomic analysis reveals a novel mitochondrial isoform of human rTS protein and unusual phylogenetic distribution of the rTS gene. BMC Genomics. $2005 ; 6: 125$.

15. Verma M, Dutta SK. DNA sequences encoding enolase are remarkably conserved from yeast to mammals. Life Sci. 1994;55(12): 893-899.

16. Sakimura K, Kushiya E, Ohshima-Ichimura Y, Mitsui H, Takahashi Y. Structure and expression of rat muscle-specific enolase gene. FEBS Lett. 1990;277(1-2):78-82. 
17. Keller A, Bérod A, Dussaillant M, Lamandé N, Gros F, Lucas M. Coexpression of alpha and gamma enolase genes in neurons of adult rat brain. J Neurosci Res. 1994;38(5):493-504.

18. Schmechel DE, Brightman MW, Marangos PJ. Neurons switch from non-neuronal enolase to neuron-specific enolase during differentiation. Brain Res. 1980;190(1):195-214.

19. Tanaka M, Sugisaki K, Nakashima K. Purification, characterization, and distribution of enolase isozymes in chicken. $J$ Biochem. 1985;98(6):1527-1534.

20. Tracy MR, Hedges SB. Evolutionary history of the enolase gene family. Gene. 2000;259(1-2):129-138.

21. Duquerroy S, Camus C, Janin J. X-ray structure and catalytic mechanism of lobster enolase. Biochemistry. 1995;34(39):12513-12523.

22. Lebioda L, Stec B, Brewer JM. The structure of yeast enolase at 2.25- $\AA$ resolution. An 8-fold beta + alpha-barrel with a novel beta beta alpha alpha (beta alpha)6 topology. J Biol Chem. 1989;264(7): 3685-3693.

23. Reed GH, Poyner RR, Larsen TM, Wedekind JE, Rayment I. Structural and mechanistic studies of enolase. Curr Opin Struct Biol. 1996;6(6):736-743.

24. Kornblatt MJ, Zheng SX, Lamandé N, Lazar M. Cloning, expression and mutagenesis of a subunit contact of rabbit muscle-specific (betabeta) enolase. Biochim Biophys Acta. 2002;1597(2):311-319.

25. Chai G, Brewer JM, Lovelace LL, Aoki T, Minor W, Lebioda L. Expression, purification and the 1.8 angstroms resolution crystal structure of human neuron specific enolase. J Mol Biol. 2004;341(4):1015-1021.

26. Wedekind JE, Poyner RR, Reed GH, Rayment I. Chelation of serine 39 to $\mathrm{Mg}^{2+}$ latches a gate at the active site of enolase: structure of the bis $\left(\mathrm{Mg}^{2+}\right)$ complex of yeast enolase and the intermediate analog phosphonoacetohydroxamate at 2.1-Å resolution. Biochemistry. 1994;33(31):9333-9342.

27. Poyner RR, Larsen TM, Wong SW, Reed GH. Functional and structural changes due to a serine to alanine mutation in the active-site flap of enolase. Arch Biochem Biophys. 2002;401(2):155-163.

28. Schreier B, Höcker B. Engineering the enolase magnesium II binding site: implications for its evolution. Biochemistry. 2010;49(35):7582-7589.

29. Giallongo A, Oliva D, Cali L, Barba G, Barbieri G, Feo S. Structure of the human gene for alpha-enolase. Eur J Biochem. 1990;190(3):567-573.

30. Peshavaria M, Day IN. Molecular structure of the human musclespecific enolase gene (ENO3). Biochem J. 1991;275(Pt 2):427-433.

31. Thierry-Mieg D, Thierry-Mieg J. AceView: a comprehensive cDNAsupported gene and transcripts annotation. Genome Biol. 2006; 7 Suppl 1: S12.1-S12.14

32. Feo S, Arcuri D, Piddini E, Passantino R, Giallongo A. ENO1 gene product binds to the c-myc promoter and acts as a transcriptional repressor: relationship with Myc promoter-binding protein 1 (MBP-1). FEBS Lett. 2000;473(1):47-52.

33. Lopez-Alemany R, Suelves M, Diaz-Ramos A, Vidal B, MunozCanoves P. Alpha-enolase plasminogen receptor in myogenesis. Front Biosci. 2005;10:30-36.

34. Perconti G, Ferro A, Amato F, et al. The Kelch protein NS1-BP interacts with alpha-enolase/MBP-1 and is involved in c-Myc gene transcriptional control. Biochim Biophys Acta. 2007;1773(12):1774-1785.

35. Altschul SF, Altschul SF, Gish W, Miller W, Myers EW, Lipman DJ. Basic local alignment search tool. J Mol Biol. 1990;215(3):403-410.

36. Gibbs RA, Rogers J, Katze MG, et al; Rhesus Macaque Genome Sequencing and Analysis Consortium. Evolutionary and biomedical insights from the rhesus macaque genome. Science. 2007;316(5822):222-234.

37. Bovine Genome Project. 2008. Available from: http://www.hgsc.bcm. tmc.edu/projects/bovine. Accessed January 6, 2011.

38. Horse Genome Project. 2008. Available from: http://www.uky.edu/Ag/ Horsemap/. Accessed January 6, 2011.

39. Waterston RH, Lindblad-Toh K, Birney E, et al; Mouse Genome Sequencing Consortium. Initial sequencing and comparative analysis of the mouse genome. Nature. 2002;420(6915):520-562.

40. Gibbs RA, Weinstock GM, Metzker ML, et al; Rat Genome Sequencing Project Consortium. Genome sequence of the Brown Norway rat yields insights into mammalian evolution. Nature. 2004;428(6982): 493-521.
41. Mikkelsen TS, Wakefield MJ, Aken B, et al. Genome of the marsupial Monodelphis domestica reveals innovation in non-coding sequences. Nature. 2007:447(7141):167-177.

42. International Chicken Genome Sequencing Consortium. Sequence and comparative analysis of the chicken genome provide unique perspectives on vertebrate evolution. Nature. 2004;432(7018):695-716.

43. Hellsten U, Harland RM, Gilchrist MJ, et al. The genome of the western clawed frog Xenopus tropicalis. Science. 2010;328(5978):633-636.

44. Sprague J, Bayraktaroglu L, Clements D, et al. The Zebrafish Information Network: the zebrafish model organism database. Nucleic Acids Res. 2005;34(Database issue):D581-D585.

45. Kent WJ, Sugnet CW, Furey TS, et al. The human genome browser at UCSC. Genome Res. 2002;12(6):996-1006.

46. McGuffin LJ, Bryson K, Jones DT. The PSIPRED protein structure prediction server. Bioinformatics. 2000;16(4):404-405.

47. Guex N, Peitsch MC. SWISS-MODEL and the Swiss-PdbViewer: an environment for comparative protein modeling. Electrophoresis. 1997; 18(15):2714-2723

48. Kopp J, Schwede T. The SWISS-MODEL Repository of annotated three-dimensional protein structure homology models. Nucleic Acids Res. 2004;32(Database issue):D230-D234.

49. Kang HJ, Jung SK, Kim SJ, Chung SJ. Structure of human alpha-enolase (hENO1), a multifunctional glycolytic enzyme. Acta Crystallogr D Biol Crystallogr. 2008;64(Pt 6):651-657.

50. Qin J, Chai G, Brewer JM, Lovelace LL, Lebioda L. Fluoride inhibition of enolase: crystal structure and thermodynamics. Biochemistry. 2006;45(3):793-800.

51. Chandran V, Luisi BF. Recognition of enolase in the Escherichia coli RNA degradosome. J Mol Biol. 2006;358(1):8-15.

52. Larkin MA, Blackshields G, Brown NP, et al. Clustal W and Clustal X version 2.0. Bioinformatics. 2007;23(21):2947-2948.

53. Giallongo A, Feo S, Moore R, Croce CM, Showe LC. Molecular cloning and nucleotide sequence of a full-length cDNA for human alpha enolase. Proc Natl Acad Sci U S A. 1986;83(18):6741-6745.

54. Calì L, Feo S, Oliva D, Giallongo A. Nucleotide sequence of a cDNA encoding the human muscle-specific enolase (MSE). Nucleic Acids Res. 1990;18(7):1893.

55. Vollmar M, Krysztofinska E, Chaikuad A, et al. Crystal structure of human beta enolase ENOB. Protein Data Bank Entry 2XSX. 2010; unpublished.

56. Kim SC, Sprung R, Chen Y, et al. Substrate and functional diversity of lysine acetylation revealed by a proteomics survey. Mol Cell. 2006; 23(4):607-618.

57. Zhou W, Capello M, Fredolini C, et al. Mass spectrometry analysis of the post-translational modifications of alpha-enolase from pancreatic ductal adenocarcinoma cells. J Proteome Res. 2010;9(6):2929-2936.

58. Ballif BA, Carey GR, Sunyaev SR, Gygi SP. Large-scale identification and evolution indexing of tyrosine phosphorylation sites from murine brain. J Proteome Res. 2008;7(1):311-318.

59. Lamandé N, Mazo AM, Lucas M, et al. Murine muscle-specific enolase: cDNA cloning, sequence, and developmental expression. Proc Natl Acad Sci U S A. 1989;86(12):4445-4449.

60. Saxonov S, Berg P, Brutlag DL. A genome-wide analysis of $\mathrm{CpG}$ dinucleotides in the human genome distinguishes two distinct classes of promoters. Proc Natl Acad Sci U S A. 2006;103(5):1412-1417.

61. Safran M, Kaelin WG Jr. HIF hydroxylation and the mammalian oxygensensing pathway. J Clin Invest. 2003;111(6):779-783.

62. Sousa LP, Brasil BS, Silva Bde M, et al. Characterization of alphaenolase as an interferon-alpha 2 alpha 1 regulated gene. Front Biosci. 2005;10:2534-2547.

63. Taylor JM, Davies JD, Peterson CA. Regulation of the myoblast-specific expression of the human beta-enolase gene. J Biol Chem. 1995;270(6): 2535-2540.

64. Feo S, Antona V, Barbieri G, Passantino R, Calì L, Giallongo A. Transcription of the human beta enolase gene (ENO-3) is regulated by an intronic muscle-specific enhancer that binds myocyte-specific enhancer factor 2 proteins and ubiquitous G-rich-box binding factors. Mol Cell Biol. 1995;15(11):5991-6002. 
65. Su AI, Wiltshire T, Batalov S, et al. A gene atlas of the mouse and human protein-encoding transcriptomes. Proc Natl Acad Sci US A. 2004;101(16):6062-6067.

66. Keller A, Ott MO, Lamandé N, et al. Activation of the gene encoding the glycolytic enzyme beta-enolase during early myogenesis precedes an increased expression during fetal muscle development. Mech Dev. 1992;38(1):41-54.

67. Gerhard DS, Wagner L, Feingold EA, et al; MGC Project Team. The status, quality, and expansion of the NIH full-length cDNA project: the Mammalian Gene Collection (MGC). Genome Res. 2004;14(10B):2121-2127.

68. Merkulova T, Lucas M, Jabet C, et al. Biochemical characterization of the mouse muscle-specific enolase: developmental changes in electrophoretic variants and selective binding to other proteins. Biochem J. 1997;323(Pt 3):791-800.

69. Keller A, Demeurie J, Merkulova T, et al. Fibre-type distribution and subcellular localisation of alpha and beta enolase in mouse striated muscle. Biol Cell. 2000;92(7):527-535.
70. Comi GP, Fortunato F, Lucchiari S, et al. Beta-enolase deficiency, a new metabolic myopathy of distal glycolysis. Ann Neurol. 2001;50(2): 202-207.

71. Mitchell BF, Pedersen LB, Feely M, Rosenbaum JL, Mitchell DR. ATP production in Chlamydomonas reinhardtii flagella by glycolytic enzymes. Mol Biol Cell. 2005;16(10):4509-4518.

72. Morita T, Kawamoto H, Mizota T, Inada T, Aiba H. Enolase in the RNA degradosome plays a crucial role in the rapid decay of glucose transporter mRNA in the response to phosphosugar stress in Escherichia coli. Mol Microbiol. 2004;54(4):1063-1075.

73. Donoghue PC, Benton MJ. Rocks and clocks: calibrating the tree of life using fossils and molecules. Trends Ecol Evol. 2007;22(8):424-431.

74. Zhao S, Choy BS, Kornblatt MJ. Effects of the G376E and G157D mutations on the stability of yeast enolase - a model for human muscle enolase deficiency. FEBS J. 2008;275(1):97-106.

75. Hyo JK, Jung S-K, Kim SJ, Chung SJ (2008) Structure of human alpha-enolase (hENO1), a multifunctional glycolytic enzyme. Acta Crystall D. 64:651-657.

\section{Publish your work in this journal}

Open Access Bioinformatics is an international, peer-reviewed, open access journal publishing original research, reports, reviews and commentaries on all areas of bioinformatics. The manuscript management system is completely online and includes a very quick and fair

\section{Dovepress}

peer-review system. Visit http://www.dovepress.com/testimonials.php to read real quotes from published authors. 